\title{
Influence of the substrate type on CVD grown homoepitaxial diamond layer quality by cross sectional TEM and CL analysis
}

\author{
D. Araújo $^{\mathrm{a}, *}$, M.P. Alegre ${ }^{\mathrm{a}}$, A.J. García ${ }^{\mathrm{a}}$, J. Navas ${ }^{\mathrm{a}}$, M.P. Villar ${ }^{\mathrm{a}}$, E. Bustarret ${ }^{\mathrm{b}}$, P.N. Volpe ${ }^{\mathrm{b}}$, F. Omnès ${ }^{\mathrm{b}}$ \\ a Dpto. Ciencia de los Materiales, Universidad de Cádiz, 11510 Puerto Real, Cádiz, Spain \\ b Institut Néel, CNRS and UJF, av. des Martyrs 25, 38041Grenoble, France
}

\section{A R T I C L E I N F O}

Available online $\mathrm{xxxx}$

Keywords:

Diamond

Cathodoluminescence

TEM

Doping

Cross section analysis

\begin{abstract}
A B S T R A C T
To assess diamond-based semiconducting devices, a reduction of point defect levels and an accurate control of doping are required as well as the control of layer thickness. Among the analyses required to improve such parameters, cross sectional studies should take importance in the near future. The present contribution shows how FIB (focused ion beam) preparations followed by electron microscopy related techniques as TEM or CL allowed to perform analysis versus depth in the layer, doping and point defect levels. Three samples grown along the same week in the same machine with identical growth conditions but on different substrates (CVD-IIIa (110) oriented, CVD-optical grade (100) oriented and a HPHT-Ib (100) oriented) are studied. Even though A-band is observed by CL, no dislocation is observed by CTEM. Point defect type and level are shown to substantially change with respect to the substrate type as well as the boron doping levels that vary within an order of magnitude. H3 present in the epilayer grown on HPHT type of substrate is replaced by T1 and NE3 point defects for epilayers grown on the CVD type one. An increase of excitonic transitions through LO phonons is also shown to take place near the surface while only TO ones are detected deeper in the epilayer. Such results highlight the importance of choosing the correct substrate.
\end{abstract}

(c) 2011 Published by Elsevier B.V.

\section{Introduction}

Despite a large effort being dedicated during the last decades by many groups worldwide to improve homoepitaxial diamond layers quality, it did not reach levels suitable enough to use such structures in devices needing high carrier mobility, even though the large intrinsic mobility of the material should potentially deliver efficient devices. However, first encouraging results on LEDs [1] or Schottky diodes [2] have been obtained recently and represent a good motivation to follow in developing new devices as FETs. Indeed, the very large thermal conductivity of diamond $(22 \mathrm{~W} / \mathrm{cm} \cdot \mathrm{K}$, around five times that of $\mathrm{Cu}$ or $\mathrm{SiC})$ makes it very attractive for power devices [3]. According to such considerations, Balmer et al. [4] propose the fabrication of a $\delta$-doped epitaxial structure to fabricate MESFET transistor devices oriented to high velocity and high power applications.

However, in practice, the growth of such a thin layer is not straightforward as doping level up to $10^{21} \mathrm{~cm}^{-3}$ should be reached to deliver sufficient holes to the channel. Kohn et al. [3] achieved doping transitions above $25 \mathrm{~nm}$, which is too large. The experimental methodology generally used for such doping level measurements is the secondary ion mass spectroscopy (SIMS), that informs only on the depth $(z$-axis) doping profiles. Thus, no in-plane $(x-y$ axis) information is

\footnotetext{
* Corresponding author. Tel.: + 34956016 427; fax: + 34956016288. E-mail address: daniel.araujo@uca.es (D. Araújo).
}

available. Lateral doping variation can thus induce artefacts in the SIMS profile. In the present paper, alternative techniques using cross sectional focused ion beam (FIB) prepared lamella, followed by electron microscopy observation, are presented. In addition, crystalline defects can also affect carrier mobility and can be detected at the same time using such a new methodology. TEM allows to assess extended defect distribution while $\mathrm{CL}$ allows to estimate the doping levels, applying the methodology proposed by Kawarada et al. [5] and Deneuville et al. [6], and also the point defect distribution with a relative quantification by comparing their respective line intensity to that of the excitons. Concerning the doping concentration, Kawarada et al. [5] give an empiric law deduced from calibration curves on a high number of samples. The experimental approach is very accurate and rigorous; however, this study is carried out on polycrystalline samples at a temperature of $100 \mathrm{~K}$. It is well known that such kind of samples contains high boron entities at grain boundaries [7], thus not at interstitial locations and thus not active as dopant. As a result their calibration curve did not correspond to that obtained very recently by Omnès et al. [8] for homoepitaxial samples. That study was carried out on homoepitaxial sample and at a temperature of $5 \mathrm{~K}$ and the dependence of the doping on the CL intensity of the bound $\left(\mathrm{BE}^{\mathrm{TO}}\right)$ to free $\left(\mathrm{FE}^{\mathrm{TO}}\right)$ exciton ratio is also linear. The highly accurate empirical law for homoepitaxial samples is based on a very high number of samples with doping between $10^{16} \mathrm{~cm}^{-3}$ (detection limit of SIMS) and $10^{18} \mathrm{~cm}^{-3}$. The curve is deduced by comparing the excitonic replicas $\mathrm{FE}^{\mathrm{TO}} / \mathrm{BE}^{\mathrm{TO}}$ ratio obtained by low temperature $\mathrm{CL}$ to the boron concentration deduced by SIMS. The lower value is closed to the 
a

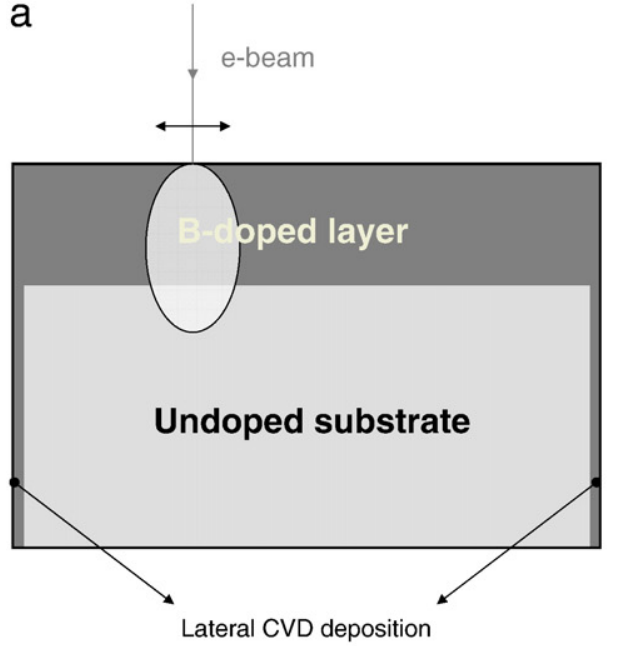

b
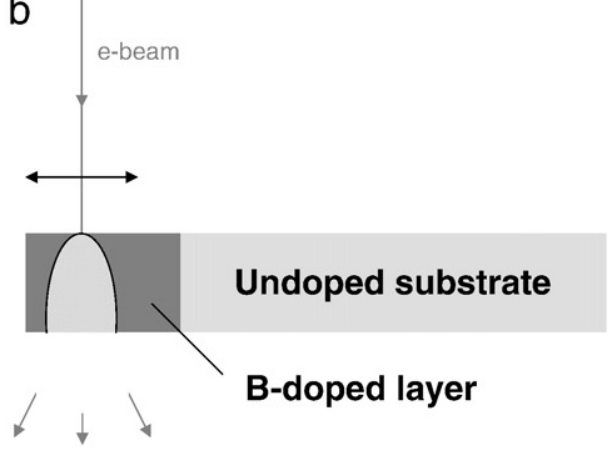

C

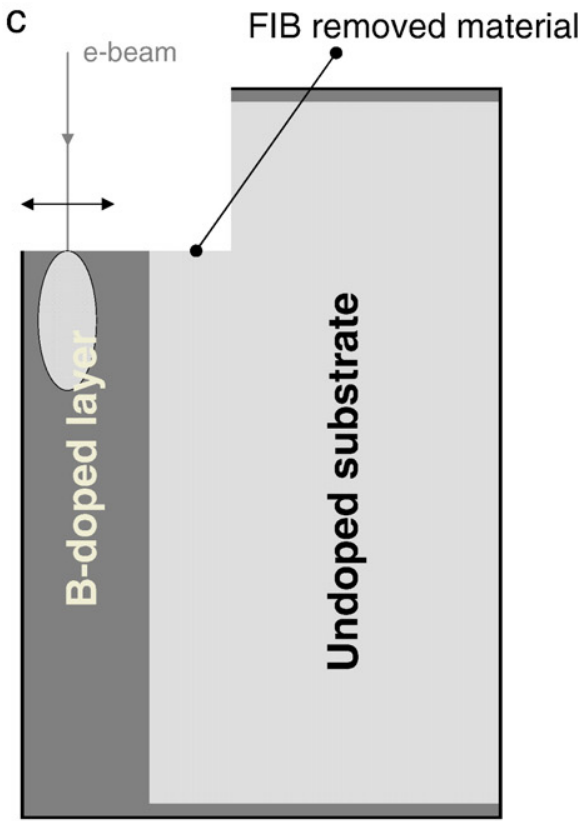

Fig. 1. Schematic description of the sample orientation where the "pear shaped" volume of interaction between high energetic incident electrons and the sample crystal is shown in each case of CL experiments. (a) Plan view orientation where the beam energy should be adjusted to generate e-h only in the epilayer. (b) Cross sectional orientation prepared by FIB assisted lift-off method to obtain a thin lamella where the incident electron can cross over the sample and then the CL signal is weaker. (c) Cross sectional orientation prepared by FIB at the edge of the sample to improve the CL signal level respect to the lamella preparation.

detection limit of the said technique while the CL peak ratio is around 1. This linear behaviour should follow down in doping level (extrapolation two order of magnitude lower is possible, sensitivity: $10^{14} \mathrm{~cm}^{-3}$ ) while for higher doping, the free exciton related peak becomes too weak to

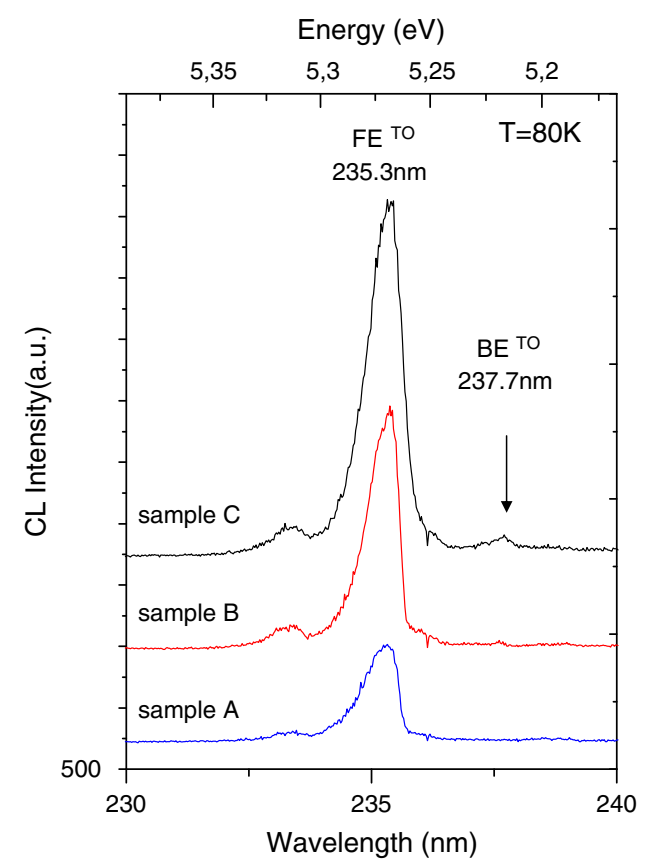

Fig. 2. CL spectra recorded at the centre of each sample in the plan view orientation at a temperature of $80 \mathrm{~K}$ and a beam energy of $10 \mathrm{keV}$. Both bound $\left(\mathrm{BE}^{\mathrm{TO}}\right)$ and free $\left(\mathrm{FE}^{\mathrm{TO}}\right)$ exciton related peak are observed for the three samples even for sample $A$, whose doping level is closed to the detection limit of the technique. The difference of their respective intensity reveals difference in doping level.

measure its intensity. But, the overlap of dopant related wave function should then diminish the bound exciton related peak energy. Indeed, Deneuville et al. [6] and more recently Ghodbane et al. [9] deduced a complementary law relating the energy shift to the doping level above $10^{19} \mathrm{~cm}^{-3}$. Both laws demonstrate the much higher sensitivity of $\mathrm{CL}$ with respect to SIMS.

\section{Experimental}

Three samples were grown by microwave plasma chemical vapour deposition (MPCVD) in a vertical silica tube reactor as described elsewhere [10] on different diamond substrates. They were grown the same week with exactly identical growing conditions that correspond to optimum parameters for low doping. The three substrates are a (110) IIIa CVD from E6 (sample A-PNV38), a (100) optical grade from E6 (sample B-PNV42), and a Ib HPHT from Sumitomo (sample C-PNV46). The growth consists of a $2 \mu \mathrm{m}$ thick epilayer (see Fig. 1a, planar view geometry for the $\mathrm{CL}$ experiments) grown at a temperature of $910{ }^{\circ} \mathrm{C}$, a pressure of 50 Torr and a $\mathrm{CH}_{4} / \mathrm{H}_{2}$ methane concentration ratio of $1 \%$ for $3 \mathrm{~h}$. The gas ratios are $[\mathrm{B}] /[\mathrm{C}]: 0.6 \mathrm{ppm}$ and $\left[\mathrm{O}_{2}\right] /\left[\mathrm{H}_{2}\right]: 0.25 \%$ (to reduce nitrogen incorporation $[11,12]$ ) and the estimate growth velocity is $800 \mathrm{~nm} / \mathrm{h}$.

For the present study, samples are prepared by the FIB-assisted lift-off technique $[13,14]$ to obtain cross section specimens (Fig. 1b). It shows how the e-beam-sample configuration is when CL is performed on an FIB prepared lamella. When the lamella is prepared for TEM observations, its thickness is around $100 \mathrm{~nm}$. Such a sample thickness was enough to detect the point defect related peaks in CL experiments but, as the excitons-related peak intensities were three orders of magnitude lower, no CL spectra with a precise location can be reported. Indeed, charging effects induce the sample to move with a velocity of around $200 \mathrm{~nm} / \mathrm{min}$ and integration of around $300 \mathrm{~s}$ was necessary to clearly distinguish between $\mathrm{FE}^{\mathrm{TO}}$ and $\mathrm{BE}^{\mathrm{TO}}$. Therefore, another experimental geometry has been purposely developed: $20 \mu \mathrm{m}$ depth was milled with the FIB just at the corner of the sample to allow a cross sectional view of the layer (see Fig. 1c). The results obtained by this method were roughly 
a

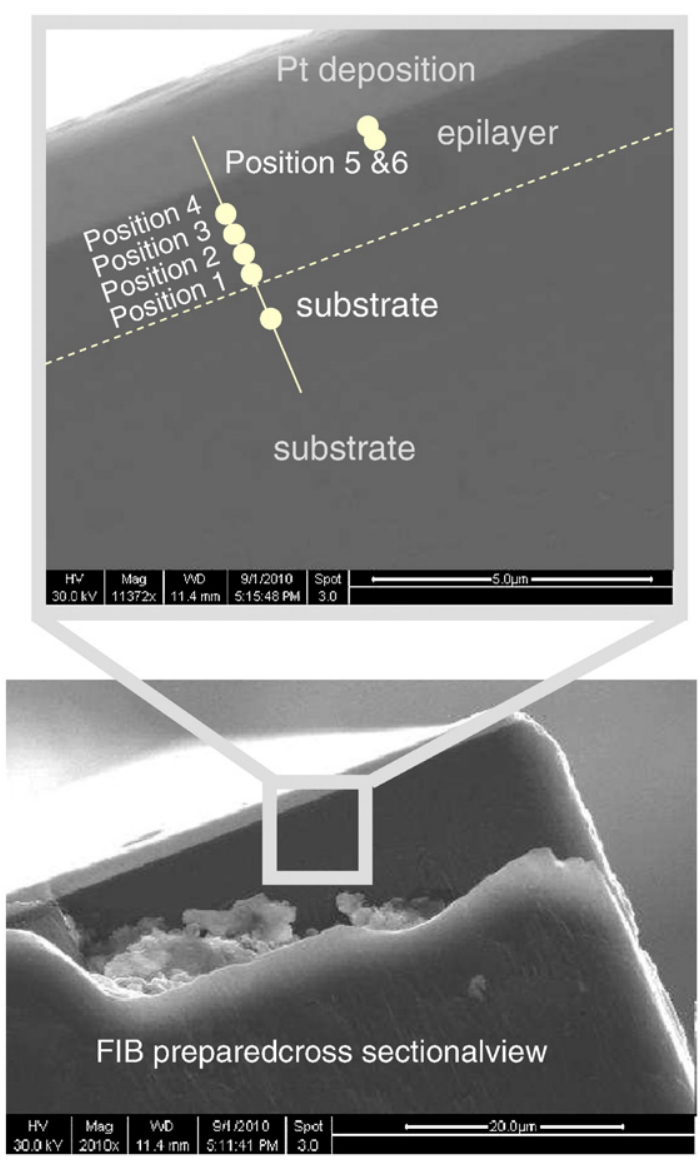

b

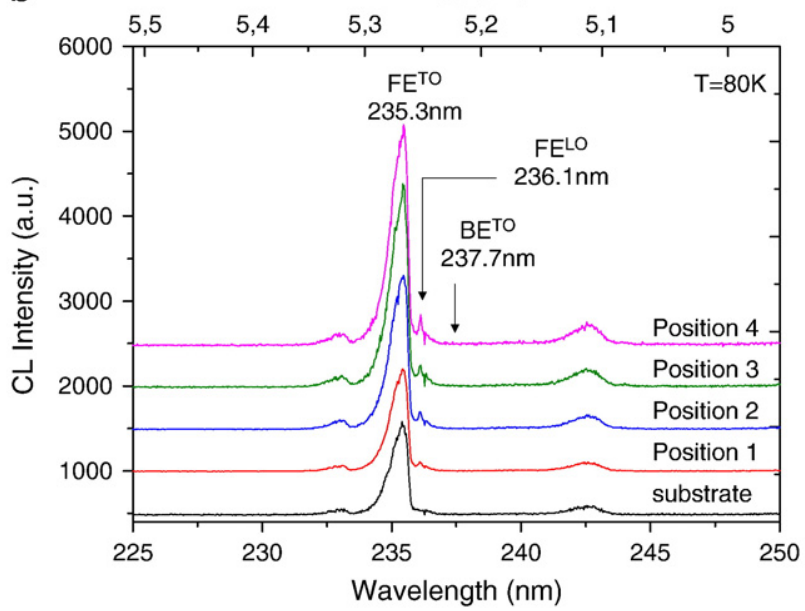

C

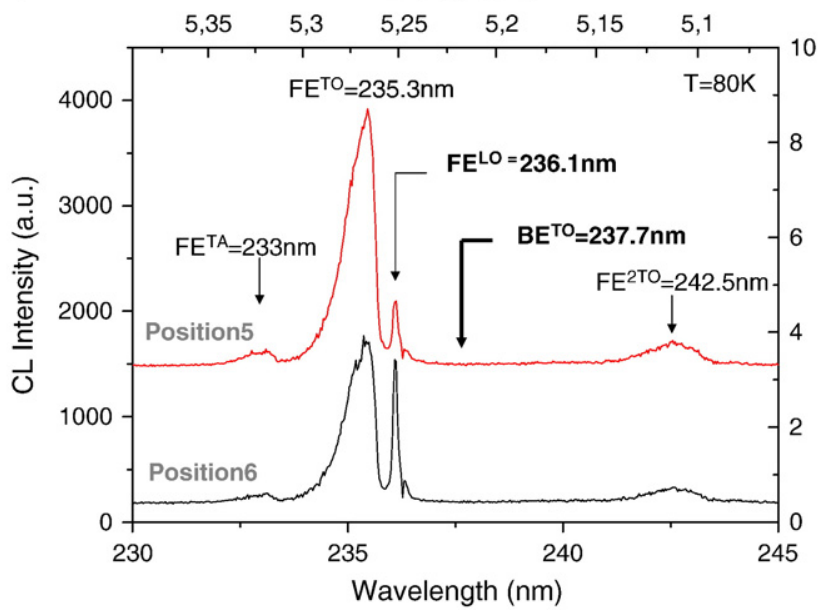

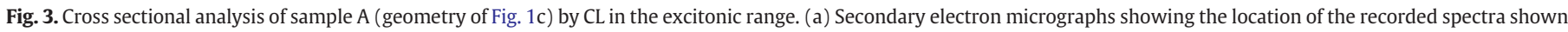

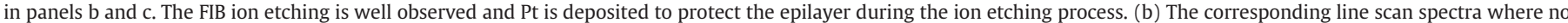

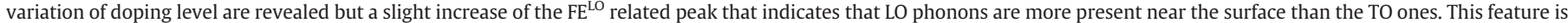
more evident in panel c. Indeed, absolute intensities can be compared as both spectra were recorded with identical conditions sequentially.

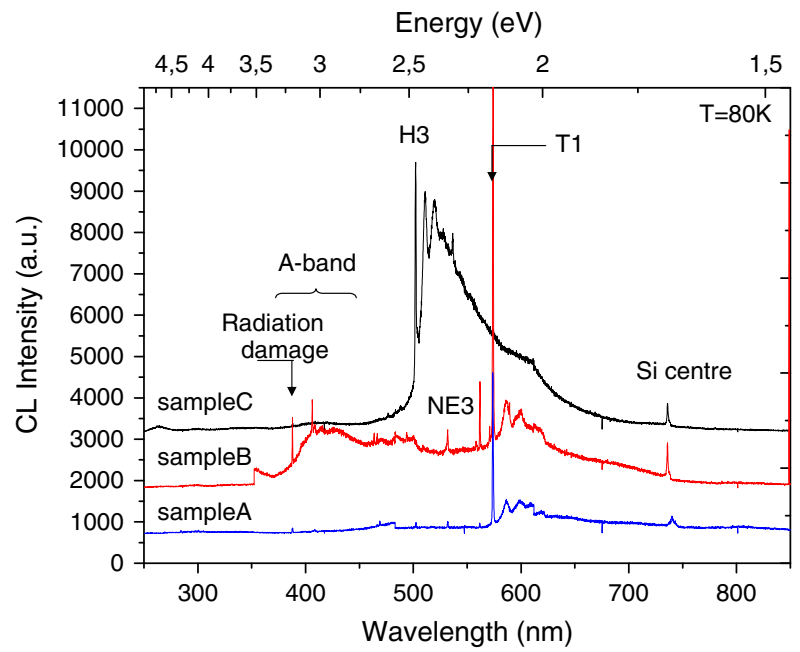

Fig. 4. CL spectra recorded at the centre of each sample in the plan view orientation at a temperature of $80 \mathrm{~K}$, a beam energy of $10 \mathrm{keV}$ and in the visible spectral range. A strong difference in the point defect type is easily observed. Note that exciting and detecting condition are identical for the three sample spectra and they were recorded sequentially, i.e., intensities can be compared. similar to those obtained on the FIB lamella but with a more accurate location of the e-beam.

TEM studies are carried out for this material. Diffraction contrast (DC) measurements are performed on a Jeol 1200EX, operating at $120 \mathrm{keV} . \mathrm{CL}$ measurements are performed on a FEI Quanta200 at $10 \mathrm{keV}$ for the planar view studies and at $30 \mathrm{keV}$ for the cross sectional ones. Temperature of $\mathrm{CL}$ experiments is $77 \mathrm{~K}$ and spectra are recorded on a Jobin-Yvon HR 250 spectrometer with 1200 lines grating. The spectrometer entrance slits are adjusted to $0.1 \mathrm{~mm}$ to clearly separate the two free and bound excitons.

\section{Doping behaviour}

First, planar view analysis at different beam energies were carried out. The beam energy has been reduced (see geometry Fig. 1a) as low as no variation between the $\mathrm{BE}^{\mathrm{TO}} / \mathrm{FE}^{\mathrm{TO}}$ ratio is observed. This value corresponds to $15 \mathrm{keV}$, that seems reasonable as the size diameter of the pear shaped volume of interactions between the incident high energetic electrons and the diamond should be less than $2 \mu \mathrm{m}$. This ensures an excitation exclusively in the epilayer; therefore, the spectra shown in the present contribution are recorded at $10 \mathrm{keV}$. In Fig. 2, the excitation was located at the centre of the sample and in Fig. 3, at the corner, used for the cross sectional analysis (see geometry of Fig. 1c). Comparison of the excitonic behaviour of the CL spectra between the three samples is reported in Fig. 2. Spectra are recorded sequentially and under identical 
a

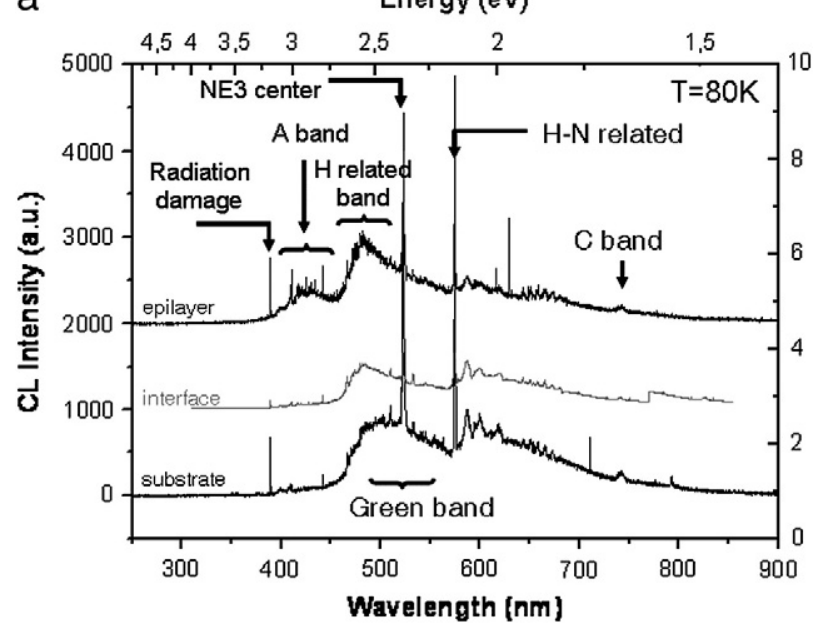

b

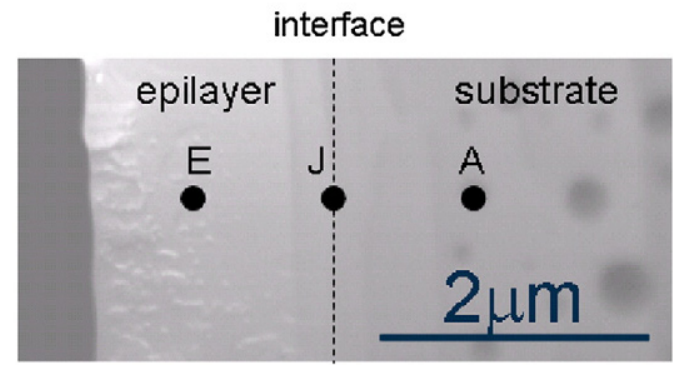

Fig. 5. (a) Cross sectional analysis of sample B on an FIB prepared lamella (geometry of Fig. 1b) by CL in the visible range. A-band is shown to increase with the height in the epilayer. (b) TEM micrograph recorded in 220 dark field conditions indicates the location where the spectra of panel a were recorded and shows also the absence of dislocations despite $\mathrm{A}$-band is recorded in the point $\mathrm{E}$.

conditions and the total peak intensities are fully reproducible. Then total intensities can also be compared. Corresponding to the observed variation of the bound and free exciton related peak intensities, a relevant variation of the doping level is then deduced. The measured $\mathrm{BE}^{\mathrm{TO}} / \mathrm{FE}^{\mathrm{TO}}$ intensity ratios are $0.01,0.038$ and 0.025 for samples $\mathrm{A}, \mathrm{B}$ and $\mathrm{C}$, respectively. The Omnès et al. [8] relation used to quantify the doping from CL spectra recorded at $5 \mathrm{~K}$ is

$$
\text { (at } T=5 \mathrm{~K}) \quad p=3.0 \cdot 10^{16} \frac{I^{\mathrm{BE}} \frac{\mathrm{E}_{\mathrm{TO}}}{I^{\mathrm{E}_{\mathrm{TO}}}}}{}
$$

Using some of the samples employed by the author to deduce this relation, a recalibration for a temperature of $80 \mathrm{~K}$ has been carried out. The empirical law becomes for $80 \mathrm{~K}$

$$
\text { (at } T=80 \mathrm{~K}) \quad p=2.4 \cdot 10^{17} \frac{I^{\mathrm{BE}_{\mathrm{TO}}}}{I^{\mathrm{FE}_{\mathrm{TO}}}}
$$

Using the this relation, doping is estimated to be $3.0 \cdot 10^{15} \mathrm{~cm}^{-3}$, $1.2 \cdot 10^{16} \mathrm{~cm}^{-3}, 7.5 \cdot 10^{15} \mathrm{~cm}^{-3}$, respectively. This points out that identical growing conditions can lead to different doping levels depending on the substrate quality. Indeed, samples A and B are CVD substrates, while sample $C$ is obtained by HPHT procedure. To investigate if a change along the epilayer height occurs, cross sectional analysis was carried out. Fig. 3a shows the experimental configuration after the FIB milling for sample A. A line scan of spectra is recorded in the cross section geometry (see Fig. 1c), and the location of the corresponding spectra shown in Fig. $3 \mathrm{~b}$ is indicated. The doping level changes are below the detection limit with respect to that reported in Fig. 2. However, an increase of the $\mathrm{FE}^{\mathrm{LO}}$ peak is revealed when the beam draws near the surface. Indeed, in Fig. 3c corresponding to positions 5 and 6 very close to the surface, this peak is detected as much important as the $\mathrm{FE}^{\mathrm{TO}}$ one. This can be easily understood by the larger presence of longitudinal phonons near the surface. In general, the Fig. $3 \mathrm{~b}$ shows that the doping is nearly constant along the height of the epilayer and that only LO replica depends on the e-beam excitation location.

\section{Extended and point defects}

The observed difference in doping level motivates the study of defects in the epilayers. Indeed, a possible relationship can exist between defects and facility of boron to be incorporated in substitutional locations of the diamond lattice. In Fig. 4, a comparison of the point defect related $\mathrm{CL}$ emission between the three samples is reported for the planar view geometry at $10 \mathrm{keV}$ (excitation exclusively in the epilayer). Strong difference between the three samples is surprisingly observed. Sample A mainly shows recombination through $\mathrm{T} 1$ centres. This peak, observed at $575 \mathrm{~nm}(2.156 \mathrm{eV})$, is generally observed in undoped CVD diamond layer (boron suppresses its intensity) and is due to nitrogen incorporation [15]. The Si centre is observed on the three samples at $737.5 \mathrm{~nm}$ $(1.681 \mathrm{eV})$ and is probably due to the reactor wall contamination during growth. The T1 peak related luminescence is also strongly observed in sample B, in addition to a weak NE3 related luminescence and a relatively strong A-band. However, the main feature of this figure is the totally different luminescence of sample $C$ with respect to samples $A$ and B. It seems that the growth on a HPHT substrate induces point defects different from the CVD ones, independently of the substrate orientation. Indeed, in sample $\mathrm{C}$, the $\mathrm{H} 3$ centre clearly dominates the spectral $\mathrm{CL}$ emission and this corresponds to the behaviour generally observed on other CVD-grown low doped diamond epilayer on HPHT substrates [16].

To investigate more deeply on the CL behaviour of the sample B epilayer, cross sectional analysis was carried out using the geometry of Fig. 1b. Spectra of Fig. 5a correspond to such a cross sectional CL emission on an FIB-lamella corresponding to the centre of the sample. Despite the location of the Fig. 5a spectra being different from those of Fig. 4, the general behaviour is confirmed. Thin lines at 3.188, 2.369, 2.154, and $1.911 \mathrm{eV}$ are observed, in addition to the large band at 2.9 (Aband), $2.5 \mathrm{eV}$, and $2.3 \mathrm{eV}$ (green band). The 2.9 band is usually labelled Aband and indicates the presence of dislocations or $\mathrm{sp}^{2}$ bonds. In the present case, this band is not present in the substrate or at the interface but increases in the epilayer. As no dislocations are evidenced by TEM (see Fig. $5 \mathrm{~b}$ ), this band is then attributed to $\mathrm{sp}^{2}$ bonds. This behaviour is corroborated by the other peaks observed at 3.188 and $2.154 \mathrm{eV}$, usually observed on irradiated, ion implanted samples (labelled in some cases as $\mathrm{T} 1$ centre) or at grain boundaries of polycrystalline diamond. It seems to be induced by complexes involving $\mathrm{H}$ and $\mathrm{N}$ atoms as for the NE3 centre observed at $2.369 \mathrm{eV}$. This indicates that nitrogen and hydrogen have been incorporated during the CVD growth and also in the substrate [15]. Finally, the C-band, that consists in a broad structured band, induced by the GR1 centre, is also present in slightly boron doped material after irradiation or near dislocations. This means, in summary, that all those peaks correspond to point defects involving boron, hydrogen and some of them also nitrogen, indicating that growth parameters should be improved to optimise doping. The TEM micrograph of Fig. 5b corresponds to 220 dark field conditions that should reveal the presence of dislocations. The presence of the A-band motivates such observations but no evidence of dislocations is found. The dark circles (see arrow) correspond to Ga aggregates generated during the TEM observation. The longer the observation time is, the larger those black circles become. EDX (X-ray emission spectroscopy) measurements corroborate it. This indicates that Ga has been incorporated during the FIB-lamella fabrication. Then, under the e-beam, Ga atoms diffuse and form such droplets. As no additional CL peaks, with respect to plan view observations, are observed, this Ga incorporation seems not to perturb the observations. After such a TEM observation, the A-band observed on the same lamella could not therefore be originated by dislocations. Then, $\mathrm{sp}^{2}$ bonds probably associated with the presence of high density of point defects should originate this A-band luminescence. 


\section{Conclusions}

The diamond epitaxial growths on different substrate types are strongly different in terms of crystalline quality (point defects) and doping level. $\mathrm{H} 3 \mathrm{CL}$ related peaks are shown to dominate the CL spectrum for epilayers grown on HPHT substrate while T1 and NE3 dominate for growth on CVD substrates independently of the substrate orientation. This change in the point defect type seems not to be responsible of the change in boron doping levels. Indeed, the growth on HPHT substrate shows a doping level different from that observed on the other growth on CVD substrate. In this case, the crystalline orientation should also play an important role. The analyses are also performed on cross sectional orientation and no significant change in doping level and point defect density is revealed. The TEM analysis certifies the absence of dislocation despite A-band being observed in the epilayer grown on the CVD (001) substrate and $\mathrm{sp}^{2}$ bonds should be responsible for this $\mathrm{CL}$ emission detected exclusively on that sample.

\section{Acknowledgements}

This work has been performed using the Electron Microscopy Facilities of the University of Cádiz (Spain). Authors thank the financial support of the Ministerio de Ciencia e Innovación of Spain (MICINN) under the project TEC2009-11399.

\section{References}

[1] V.C. Coffey, Laser Focus Word 37 (2001) 50

[2] T. Makino, S. Tanimoto, H. Kato, N. Tokuda, M. Ogura, D. Takeuchi, K. Ogama, H. Ohashi, H. Okushi, S. Yamasaki, Phys. Status Solidi A 206 (2009) 2086

[3] E. Kohn, A. Denisenko, Thin Solid Films 515 (2007) 4333.

[4] R.S. Balmer, I. Friel, S.M. Woollard, C.J.H. Wort, G.A. Scarsbrook, S.E. Coe, H. El-Hajj, A. Kaiser, A. Denisenko, E. Kohn, J. Isberg, Philos. Trans. R. Soc. A 366 (2008) 251.

[5] H. Kawarada, H. Matsuyama, Y. Yokota, T. Sogi, A. Yamaguchi, A. Hiraki, Phys. Rev. B 47 (1993) 3633

[6] A. Deneuville, C. Baron, S. Ghodbane, C. Agnes, Diamond Relat. Mater. 16 (2007) 915

[7] N. Dubrovinskaia, R. Wirth, J. Wosnitza, T. Papageorgiou, H.F. Braun, N. Miyajima L. Dubrovinsky, PNAS 105 (2008) 11619.

[8] F. Omnès, P.R. Muret, P.N. Volpe, M. Wade, J. Pernot, F. Jomard, poster P2.62 at the Diamond 2010 Conference in Budapest (5-9 September), in press. (2011).

[9] S. Ghodbane, F. Omnès, C. Agnes, Diamond Relat. Mater. 19 (2010) 273.

[10] T. Klein, P. Achatz, J. Kacmarcik, C. Macenat, F. Gustafsson, J. Marcus, E. Bustarret, J. Pernot, F. Omnès, BoE. Sernelius, C. Person, A. Ferreira da Silva, C. Cyterman, Phys. Rev. B 75 (2007) 165313.

[11] D. Araujo, M. Kadri, M. Wade, E. Bustarret, A. Deneuville, Phys. Status Solidi C 2 (2005) 1336.

[12] A.M. Kadri, D. Araujo, M. Wade, A. Deneuville, E. Bustarret, Diamond Relat. Mater. 14 (2005) 566.

[13] R.M. Langford, M. Rogers, Micron 39 (2008) 1325

[14] D. Takeuchi, H. Watanabe, S. Yamanaka, H. Okushi, H. Sawada, H. Ichinose, T. Sekiguchi, K. Kajimura, Phys. Rev. B 63 (2001) 245328.

[15] A.M. Zaitsev, Optical Properties of Diamond: A Data Handbook, Springer-Verlag, Berlin Heidelberg, 2001.

[16] M. Wade, M. Kadri, E. Bustarret, A. Deneuville*, P. Muret, D. Araujo, Phys. Status Solidi A 201 (2004) 2457. 